\title{
Évaluation de la qualité bactériologique des eaux de puits et de forage à Lomé, Togo
}

\author{
K. Soncy, B. Djeri, K. Anani, M. Eklou-Lawson, Y. Adjrah, D.S. Karou*, Y. Ameyapoh et C. de Souza \\ Laboratoire de Microbiologie et de Contrôle de qualité des Denrées Alimentaires (LAMICODA), École Supérieure des \\ Techniques Biologiques et Alimentaires (ESTBA), Université de Lomé, BP 1515, Lomé, Togo \\ ${ }^{*}$ Correspondance : simplicekarou@hotmail.com
}

Original submitted in on $12^{\text {th }}$ June 2015. Published online at www.m.elewa.org on $31^{\text {st }}$ July 2015 http://dx.doi.org/10.4314/jab.v91i1.6

\section{RÉSUMÉ}

Objectif: Le but de cette étude était d'évaluer la qualité microbiologique des eaux de puits et de forage collectées dans la ville de Lomé.

Méthodologie : Au total, 207 échantillons d'eau de puits et de 197 échantillons d'eau de forages de la ville de Lomé collectés entre juin 2012 et juillet 2013 ont été analysés en utilisant les méthodes normalisées de routine de l'Association Française de Normalisation (AFNOR).

Résultats et conclusion: Les résultats ont montré une non-conformité des eaux de puits par rapport aux germes de contamination fécale dans $65 \%$ des cas et dans $70 \%$ des cas par rapport à $E$ coli. Les eaux de forages étaient contaminées à $53,54 \%$ par la flore aérobie mésophile, à $26,77 \%$ par les coliformes totaux et à 2,03\% par les streptocoques fécaux. La présence des germes indicateurs de contamination fécale dans les eaux analysées expose les consommateurs au risque de gastro-entérites. Des mesures doivent être prises pour la surveillance et la désinfection de ces eaux avant leur utilisation.

Mots clés : Eau, puits, forage ; qualité bactériologique, Togo.

\section{Evaluation of the bacteriological quality of wells and drillings water in Lomé, Togo}

\section{ABSTRACT}

Objective: The aim of this study was to evaluate the microbiological quality of wells and drillings water collected in the city of Lomé.

Methodology: A total of 207 well water samples and 197 samples of water from drillings in the city of Lomé collected between June 2012 and July 2013 were analyzed using standard routine methods of the French Association of Standardization (AFNOR).

Results and Conclusion: The results showed non-compliance of well water related to fecal contamination germs in $65 \%$ of cases and in $70 \%$ of cases related to E. coli. The drillings waters were contaminated in cases $53.54 \%$ by mesophilic aerobic flora, in $26.77 \%$ cases by total coliforms and in $2.03 \%$ cases by fecal streptococci. The presence of indicator organisms of fecal contamination in the analyzed waters exposes consumers to the risk of gastroenteritis. Measures should be taken to monitoring and disinfection of this water before use.

Keywords: Water, wells, drilling, bacteriological quality, Togo. 


\section{INTRODUCTION}

Les besoins en eau de la population de Lomé ont été décuplés avec l'accroissement que connaît la ville depuis les deux dernières décennies. Lomé à l'instar des grandes villes africaines, connaît une démographie galopante avec une urbanisation mal contrôlée. Cette situation a favorisé l'émergence de quartiers périphériques contrastant avec le centreville. Ces quartiers souvent populeux sont installés sur des sites qui manquent de systèmes d'assainissement, d'hygiène et surtout d'infrastructures sanitaires et sociales de même que de réseau d'adduction d'eau potable. L'alimentation en eau de la ville est du ressort du Ministère en charge de l'eau. Sa mission principale est, par l'intermédiaire des structures et services qualifiés, de fournir de l'eau potable à la population des villes (La Togolaise des Eaux - TdE) et en milieu rural (La Division de l'Hydraulique Villageoise). Le réseau d'adduction d'eau potable n'a pas suivi l'extension de la ville. Cela explique les problèmes d'approvisionnement en eau qui sont observés dans ces nouveaux quartiers de Lomé. La population en réaction à la pénurie d'eau a eu recours à des puits à large diamètre surtout dans les zones où l'eau est facilement accessible ou à des forages pour accéder à l'eau au-delà de 30 mètres sous terre. Si ces ouvrages ont l'avantage de résoudre le problème de la disponibilité de l'eau, la qualité de cette denrée n'est pas souvent garantie. En 2002 une étude a révélé que les eaux

\section{MATÉRIEL ET MÉTHODES}

Matériel : L'échantillonnage effectué de façon aléatoire, s'est déroulé entre juin 2012 et juillet 2013. Le matériel de prélèvement et de transport est constitué des flacons stériles $\left(121^{\circ} \mathrm{C} / 25 \mathrm{mn}\right)$ de $500 \mathrm{ml}$, d'une glacière munie d'éléments réfrigérants et d'un bruleur pour stériliser les robinets avant les prélèvements. Le seau de puisage de chaque maison est utilisé pour prélever les eaux de puits. Les prélèvements d'eau ont été réalisés au niveau de 207 puits et de 197 forages pris au hasard dans la ville de Lomé. Les milieux de culture et les germes recherchés sont consignés dans le tableau 1. de puits dans la zone Sud Lagunaire de Lomé étaient contaminées par des Coliformes thermotolérants et Escherichia coli (SADAOC, 2002). D'après l'Organisation Mondiale de la Santé, l'eau destinée à la consommation et aux besoins des ménages ne doit pas contenir de microorganismes pathogènes ; aucun échantillon de 100 $\mathrm{ml}$ d'une eau destinée à la consommation ne doit contenir de germes anaérobies sulfito-réducteurs, de coliformes et de streptocoques (WHO, 2008). Toutes les eaux de puits analysées par Makoutode et al. (1999) à Porto Novo étaient contaminées par des Entérocoques et $31 \%$ d'entre elles renfermaient $E$. coli. Ces bactéries témoins de contaminations d'origine fécale des eaux ont été également retrouvées dans les eaux de puits et de forages de certains quartiers de Bangui (Mokofio et al., 1991) et de Bamako (Coulibaly, 2005). Selon Chippaux et al. (2002), l'origine des pollutions peut être attribuée aux défauts d'assainissement et de collecte des ordures ménagères, au transfert de polluants à partir des couches superficielles du sol, aux conditions de puisage et à la structure des ouvrages. La présente étude a eu pour objectif l'évaluation de la contamination bactérienne des eaux de puits et de forages de la ville de Lomé afin de mesurer les risques sanitaires auxquels sont exposées les personnes qui les utilisent pour leurs besoins.

Méthodes : Les méthodes normalisées de routine de l'Association Française de Normalisation (AFNOR) ont été utilisées pour la recherche et le dénombrement des germes contaminant des eaux (Tableau 1). La méthode par incorporation dans la gélose a été utilisée pour la recherche et le dénombrement de la flore mésophile totale (FMT), les coliformes totaux, les coliformes thermotolérants, et les streptocoques fécaux. Escherichia coli a été recherché en utilisant le milieu Brillance $^{\mathrm{TM}}$ E. coli (Code CM1046, OXOID) selon les prescriptions de la fiche technique du milieu. Les autres milieux de culture sont de Bio Rad (France). 
Tableau 1 : Germes recherchés et méthodes de dénombrement dans les eaux analysées

\begin{tabular}{|l|l|l|l|}
\hline Germes recherchés & Milieux de culture & Méthodes normalisées & Température/Temps d'incubation \\
\hline Flore mésophile totale & PCA & NF V08-051, Fév. 1999 & $30^{\circ} \mathrm{C} / 72 \mathrm{~h}$ \\
\hline Coliformes totaux & VRBL & NF V08-050, Déc. 1992 & $30^{\circ} \mathrm{C} / 24 \mathrm{~h}$ \\
\hline Coliformes thermotolérants & VRBL & NF V08-016, Déc. 1992 & $44^{\circ} \mathrm{C} / 24 \mathrm{~h}$ \\
\hline Escherichia coli & Brillance E. coli & $\begin{array}{l}\text { (CM 1046) Brilliance }{ }^{T M} \text { E. } \\
\text { coli/coliform selective agar }\end{array}$ & $37^{\circ} \mathrm{C} / 24 \mathrm{~h}$ \\
\hline Streptocoques fécaux & Slanetz et Bartley & NF T 90-416, Oct. 1985 & $37^{\circ} \mathrm{C} / 48 \mathrm{~h}$ \\
\hline $\begin{array}{l}\text { Anaérobies sulfito- } \\
\text { réducteurs }\end{array}$ & TSN & XP V08-061, Av. 1996 & $44^{\circ} \mathrm{C} / 48 \mathrm{~h}$ \\
\hline
\end{tabular}

PCA : Plate Count Agar ; VRBL : Gélose au Cristal Violet au Rouge Neutre à la Bile et au Lactose ; TSN : Tryptone Sulfite Néomycine.

Les boîtes de milieu de culture ont été incubées aux températures respectives indiquées dans le tableau 1. Les anaérobies sulfito-réducteurs (ASR) ont été recherchés en introduisant $1 \mathrm{ml}$ de l'inoculum dans 19 $\mathrm{ml}$ de gélose TSN (Tryptone Sulfite Néomycine) préparée dans des tubes à essai. L'incubation est faite à $44^{\circ} \mathrm{C}$ pendant $48 \mathrm{~h}$. Les résultats ont été appréciés par rapport aux Critères de l'Union Européenne (Directive du conseil 98/83/EC). Au cours des prélèvements, l'environnement des puits et des maisons a été

\section{RÉSULTATS}

État sanitaire de l'environnement dans la zone de prélèvement : L'observation de l'environnement immédiat des puits a permis de constater que les puits dont les eaux ont été prélevées pour cette étude sont traditionnels. Ils ont été réalisés pour la plupart par des puisatiers locaux disposant d'un matériel rudimentaire. Ces puits ont environ $0,85 \mathrm{~m}$ de diamètre ; la profondeur de l'eau varie entre 0,4 et $15 \mathrm{~m}$. Presque tous ces puits disposaient d'une margelle non couverte (sauf 27/207) de 0,5 à $1 \mathrm{~m}$ de hauteur par rapport au niveau du sol. Les sanitaires constitués de fosses septiques et de puisards à fond perdu, étaient situés dans un rayon de $20 \mathrm{~m}$ au maximum des puits. Les puits ont été pour la plupart réalisés dans des zones sableuses où la nappe est moins profonde. Les forages visités dans la présente étude sont des structures relativement protégées, constituées de puits de diamètre variant entre 15 et $20 \mathrm{~cm}$. Ils sont équipés de pompe immergée et recouverts pour la plupart d'une petite dalle en béton. L'eau de forage est recueillie dans un polytank d'où elle sera ensuite distribuée par des tuyaux en PVC. L'eau de forage est destinée à tous les usages. L'eau de puits d'après les utilisateurs n'est pas utilisée pour la boisson. Pour ce qui est des eaux usées des ménages visitées, elles sont déversées soit directement dans la cour ou dans la rue devant les maisons. Des animaux apprécié. Des données sur l'état des puits et des forages ont été également consignées sur une fiche d'enquête.

Analyse statistique : Les résultats ont été analysés par SYSTAT 11. L'analyse de la variance a été effectuée par ANOVA (One-way analysis of variance). La différence entre les échantillons a été déterminée par le test Tukey's multiple comparaison, par un coefficient de sécurité de $95 \%$ et un degré de liberté au risque de $5 \%$.

domestiques en divagation sont observés dans certaines maisons.

Qualité microbiologique des eaux analysées : Les résultats des analyses microbiologiques ont montré que les eaux de puits étaient toutes contaminées par la plupart des germes recherchés. Elles ont été fortement contaminées par la flore mésophile totale et les germes de contamination fécale. La contamination des eaux de puits par la FMT a induit plus de $99 \%$ de non-conformité soit deux puits seulement présentant des valeurs en dessous de celles fixées par les critères d'évaluation (FMT $=100 / \mathrm{ml})$. Les coliformes totaux, coliformes thermotolérants et $E$. coli ont induit respectivement $98,55 \%, \quad 86,48 \%$ et $79,08 \%$ d'échantillons non conformes aux critères (Figure 1). Les Streptocoques fécaux ont été retrouvés dans $65,7 \%$ des échantillons d'eau de puits analysés. Les germes ASR ont induit $31,73 \%$ de non-conformité par rapport aux échantillons des eaux de puits. Les eaux de forages ont été moins contaminées. Tous les échantillons (197/197) ont été conformes par rapport aux coliformes thermotolérants, E. coli et le germes ASR (Tableau 2). Les échantillons analysés ont été non-conformes par rapport aux coliformes totaux $(26,72 \%)$, streptocoques fécaux $(2,03 \%)$ et la FMT $(53,54 \%)$. Le rapport de charge microbienne moyenne est de l'ordre de 18,6 et 42,6 fois 
$\begin{array}{lll}\begin{array}{l}\text { Souncy et al. J. Appl. Biosci. Évaluation de la qualité bactériologique des eaux de puits et de forage } \\ \text { à Lomé, Togo }\end{array} & \text { J }\end{array}$ entre les deux types d'eau par rapport à la FMT et aux coliformes totaux.

Tableau 2 : Évaluation de la qualité des eaux analysées

\begin{tabular}{|l|l|l|l|l|l|}
\hline \multirow{2}{*}{ Germes recherchés } & \multicolumn{2}{l|}{$\begin{array}{l}\text { Valeurs extrêmes } \\
\text { en UFC/ml }\end{array}$} & \multicolumn{2}{l|}{ Critères $^{*}$} & \multicolumn{2}{l|}{$\begin{array}{l}\text { Nombre de résultats } \\
\text { Satisfaisants }\end{array}$} \\
\cline { 2 - 7 } & Puits & Forages & $\mathbf{M}$ & $\begin{array}{l}\text { Puits } \\
\mathbf{n}=\mathbf{2 0 7}\end{array}$ & $\begin{array}{l}\text { Forages } \\
\mathbf{n}=197\end{array}$ \\
\hline Flore mésophile totale & $\begin{array}{l}60 \\
530000\end{array}$ & $\begin{array}{l}0 \\
308427\end{array}$ & 100 & 2 & 92 \\
\hline Coliformes totaux & $\begin{array}{l}0 \\
6330\end{array}$ & $\begin{array}{l}0 \\
1720\end{array}$ & 0 & 3 & 145 \\
\hline Coliformes thermotolérants & $\begin{array}{l}0 \\
2500\end{array}$ & 0 & 0 & 28 & 197 \\
\hline Escherichia coli & $\begin{array}{l}0 \\
700\end{array}$ & 0 & 0 & 62 & 197 \\
\hline Streptocoques fécaux & $\begin{array}{l}0 \\
700\end{array}$ & 0 & 0 & 71 & 194 \\
\hline Anaérobies sulfito-réducteurs & $\begin{array}{l}0 \\
46\end{array}$ & 0 & 2 & 142 & 197 \\
\hline
\end{tabular}

${ }^{*}$ Critères de l'Union Européenne de Directive du conseil 98/83/EC (m) ; $\mathrm{n}$ : nombre d'échantillon analysé

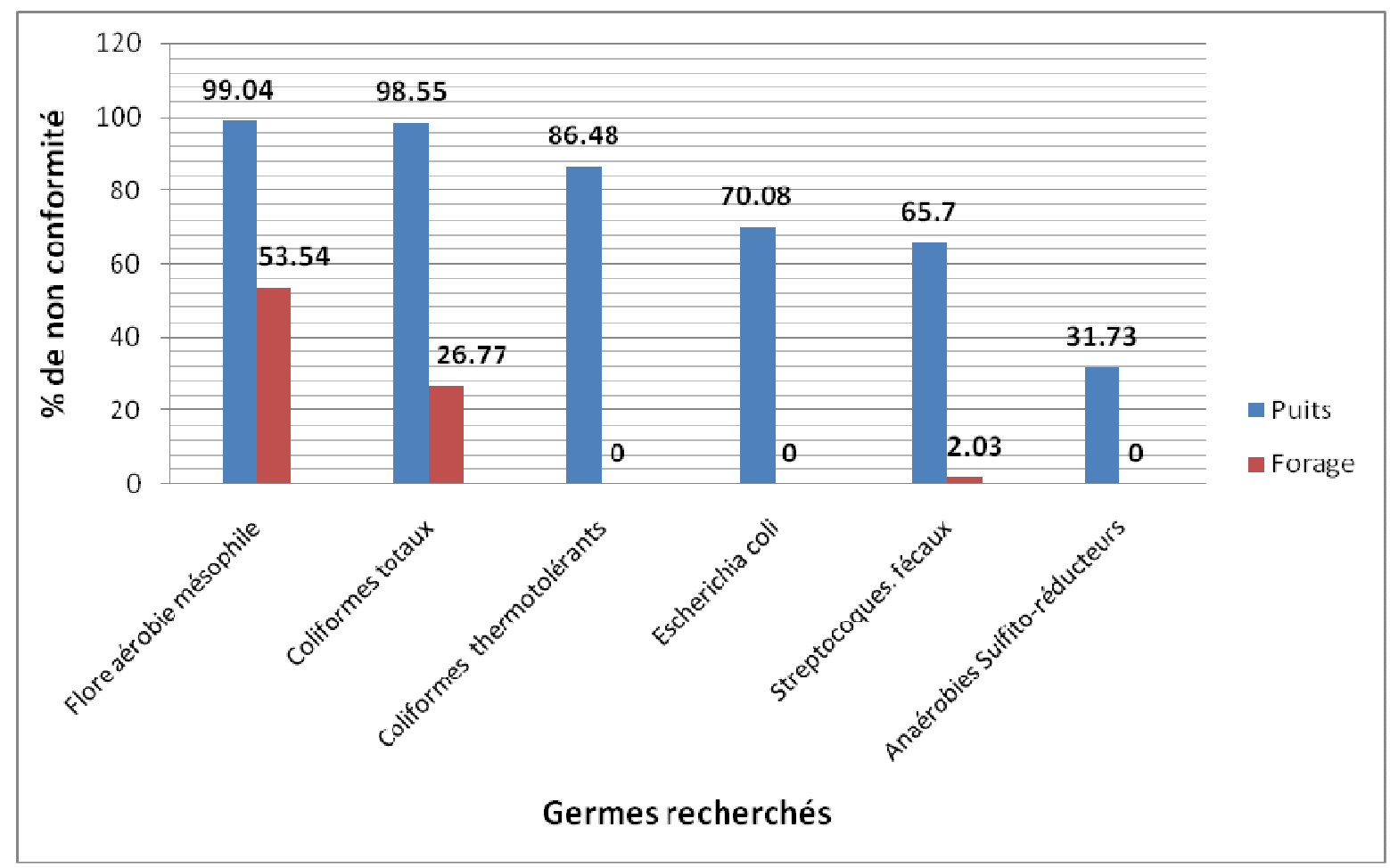

Figure 1 : Non-conformité induite par rapport aux germes recherchés.

\section{DISCUSSION}

Cette étude a eu pour but d'évaluer la qualité microbiologique des eaux de puits et de forage de la ville de Lomé. Les résultats ont indiqué que les eaux de puits analysées ont été contaminées par la plupart des germes recherchés. La contamination d'origine fécale a été importante. Elle était respectivement de 98,55\%, $86,48 \%$ et $70,08 \%$ par rapport aux Coliformes totaux, Coliformes thermotolérants et $E$. coli. Chippaux et al. 
(2002) ont aussi trouvé une contamination excessive des eaux de puits de Niamey en coliformes totaux et streptocoques fécaux. Des observations similaires à ceux des eaux de puits de Lomé ont été rapportées à Meknès au Maroc concernant les coliformes totaux et les streptocoques fécaux (Belghiti et al., 2013). A Abengourou en Côte d'lvoire, Aka et al. (2013) ont dénombré dans $28 \%$ des eaux de puits analysées des souches d'Escherichia coli. Selon Bricha et al. (2007), les réseaux d'égouts, les fosses septiques, les eaux usées des usines et les déchets solides seraient les sources principales de pollution des eaux souterraines dans le secteur urbain et dans les zones périurbaines. De plus, La contamination de la nappe des puits dépend de la perméabilité du sol, de la profondeur de la nappe, de l'absence ou l'inadaptation des ouvrages d'assainissement, de la mauvaise gestion des ordures et de la méthode de puisage (Yapo et al., 2010 ; Degbey et al., 2010 ; Coulibaly, 2005 ; Hassane, 2010). En effet, le développement rapide de la ville de Lomé a engendré des problèmes dans la gestion des eaux usées et des ordures ménagères qui étaient à l'origine de la contamination de la nappe peu profonde (moins d'un mètre en toute saison dans certains quartiers) dans laquelle sont directement déversées les eaux usées des ménages, des toilettes et des effluents de fosses septiques. La profondeur des puits ne dépasse pas pour la plupart deux mètres dans la zone sud lagunaire de Lomé. Ce problème est similaire à celui de Bangui où presque tous les puits sont souillés par les germes fécaux et où la pollution est d'autant plus importante que le quartier est situé à basse altitude (Mokofio et al., 1991). Les eaux de forage analysées sont moins contaminées par rapport à celles des puits. II existe une différence significative $(p<0,05)$ entre l'eau des puits et celle des forages par rapport à tous les germes

\section{CONCLUSION}

Les eaux de puits et de forages sont encore des sources importantes d'approvisionnement en eau à Lomé. Elles sont destinées à divers usages et surtout dans l'alimentation. La contamination de ces eaux par des bactéries d'origine fécale constitue un risque majeur de gastro-entérites pour les consommateurs. Les causes sont surtout le défaut d'assainissement et les recherchés. Cela peut être dû à l'écart entre la profondeur des puits et celle des forages. En effet, les forages à Lomé sont réalisés dans les quartiers où il est plus difficile aux habitants de réaliser des puits à large diamètre. A cause du relief et la nature (argile sablonneux)du sol, l'accès à l'eau dans cette zone se fait par des forages à des profondeurs

qui vont en moyenne au-delà de 30mètres. Malheureusement, l'eau de puits intervient encore pour une grande part dans la consommation en eau des populations de la ville de Lomé. Même si une ménage est branché sur le réseau d'adduction de la ville dont l'eau a été jugée de bonne qualité bactériologique par Kimassoum et al. (2011), cette eau n'est utilisée que pour la boisson et des fois pour la préparation des repas. L'eau de puits, si elle est facilement accessible, est utilisée pour la vaisselle, pour laver les habits et faire les toilettes. La qualité bactériologique des eaux des puits met en évidence la contamination d'origine fécale de la nappe phréatique spécialement par $E$. coli (Degbey et al., 2010). Ces germes ont été dénombrés dans les jus frais obtenus à partir des fruits lavés à l'aide des eaux de puits de Lomé (Soncy et al., 2014). Or, I'Institut national de santé publique du Québec (2004) a recommandé que l'eau contaminée par la bactérie de $E$. coli ne soit pas consommée, à moins qu'elle ait été bouillie pendant au moins une minute. De plus il faudrait également s'abstenir de préparer des glaçons, de laver les aliments, de se brosser les dents ou de donner le bain à un bébé avec cette eau. De leurs cotés, Bawa et al (2008) et Tampo et al (2014) proposent une chloration adaptée à la composition des eaux de puits et de forages de Lomé pour les rendre potables afin de compenser l'insuffisance du réseau d'adduction d'eau et de réduire les risques de maladies hydriques.

mauvaises habitudes dans la gestion de l'hygiène dans les maisons. II en ressort que l'eau des puits de Lomé sont très fortement polluées par tous les germes recherchés avec plus de $70 \%$ de puits contenant Escherichia coli. L'eau de forage est relativement moins contaminée mais avec près de $26 \%$ de non-conformité par rapport aux coliformes totaux.

\section{RÉFÉRENCES BIBLIOGRAPHIQUES}

Aka N, Bamba SB, Soro G, Soro N, 2013. Étude hydro chimique et microbiologique des nappes d'altérites sous climat tropical humide : Cas du département d'Abengourou (Sud-Est de la Cote

d'Ivoire) Larhyss Journal, ISSN 1112-3680, $n^{\circ} 16$, pp. 31-52.

Bawa ML, Tchacala I, Djanéyé-BouindjouG, 2008. Détermination de la demande en chlore des 
eaux de puits et de forages d'un quartier périphérique de la ville de Lomé : Incidence sur la désinfection. Journal des Sciences et Technique, Vol. $7 \mathrm{n}^{\circ} 2$ pp. 19-24.

Belghiti ML, Chahlaoui A, Bengoumi D, El Moustaine R, 2013. Étude de la qualité physico-chimique et bactériologique des eaux souterraines de la nappe plio-quaternaire dans la région de Meknès (Maroc) Larrhyss Journal, $n^{\circ} 14, p p$. 21-36.

Boubakar Hassane A, 2010. Aquifères superficiels et profonds et pollution urbaine en Afrique : Cas de la communauté urbaine de Niamey (NIGER), Thèse de l'Univ, Abdou Moumouni de Niamey (Niger), 198p.

Bricha S, Ounine K, Oulkheir S, El Haloui N, Attarassi B, 2007. Étude de la qualité physico-chimique et bactériologique de la nappe phréatique de M'nasra, Maroc. Afrique Science 03(3), 391404.

Chippaux J-P, Houssier S, Gross P, Bouvier C, Brissaud $F$, 2002. Étude de la pollution de l'eau souterraine de la ville de Niamey, Niger. Bull Soc Pathol Exot, 2002 94, 2, 119-123.

Coulibaly K, 2005. Étude de la qualité physico-chimique et bactériologique de l'eau de puits de certains quartiers du district de Bamako ; Thèse de Doctorat en Pharmacie, Faculté de Médecine de Pharmacie et d'Odonto-Stomatologie, Université de Bamako, pp69.

Degbey C, Makoutode M, de Brouwer C, 2010. La qualité de l'eau de boisson en milieu professionnel à Godomey en 2009 au Bénin Afrique de l'Ouest, J Int Santé Trav 2010 ; 1 $: 15-22$.

Institut national de santé publique du Québec, 2004. Étude du risque de gastro-entérite chez les familles utilisant l'eau d'un puits domestique ; Étude sur la qualité de l'eau potable dans sept bassins versants en surplus de fumier et impacts potentiels sur la santé. Direction Risques Biologiques, Environnementaux et Occupationnels Institut National de Santé Publique du Québec et Unité de Recherche en Santé Publique Centre de Recherche du CHUL (CHUQ).

Kimassoum D, Tidjani A, Doutoum AA, Ameyapoh $Y$, Soncy K, Dossou K, Anani K, de Souza C, 2011. Évaluation de la qualité hygiénique de l'eau de robinet produite par la Société Togolaise des Eaux (TdE) : cas de neuf quartiers de la Commune de Lomé (Togo) Microbiol. Hyg. Alim - Vol 23, n68.

Makoutode M, Assani AK, Ouendo E-M, Agueh VD, Diallo O, 1999. Qualité et mode de gestion des eaux de puits en milieu rural au Bénin : cas de la sous -préfecture de Grand-Popo. Médecine d'Afrique Noire : 46 (11).

Mokofio F, Renaudet J, Opandy C, Bastard G, Abeye J, Yete ML, Touabe J, Gondao L, Vohito JA, 1991. Qualité bactériologique de l'eau des puits, des sources et de forages dans la ville de Bangui : Premiers Résultats et Perspectives. Médecine d'Afrique Noire : 38(11).

$S A D A O C$, 2002. Hygiène alimentaire et problématique de l'alimentation de rue en Afrique de l'Ouest. Volume 6, Numéro 1, Mai 2002 ; http://www.icilome.com/ consulté le 12/04/2014.

Soncy K, Anani K, Adjrah Y, Djeri B, Eklu MM, Karou DS, Ameyapoh Y, de Souza C. 2014. Hygienic quality and nutrient characterization of three fruits juices sold in Lomé Schools. Int. J. of In. in Bio and Che. Sci., 5: 1-6.

Tampo L, Ayah M, Kodom T, Tchakala I, Boguido P, Bawa LM, Djanéyé-Bouindjou G, 2014. Impact de la demande en chlore et de la chloration sur la désinfection des eaux de puits des quartiers de Lomé : cas des quartiers de Démakpoé et d'Agbalépédogan (Togo). Journal of Applied Biosciences 75 :6272-6281.

World Health Organization, 2008. Guidelines for drinking-water quality, third edition incorporating the first and second addenda, volume1, Recommandations, Geneva.

Yapo OB, Mambo V, Seka A, Ohou MJA, Konan F, Gouzile V, Tidou AS, Kouame KV, Houenou P, 2010. Évaluation de la qualité des eaux de puits à usage domestique dans les quartiers défavorisés de quatre communes d'Abidjan (Côte d'Ivoire) : Koumassi, Marcory, Port-Bouet et Treichville Int. J. Biol. Chem. Sci. 4(2) : 289307. 